\title{
GGA $+U$ studies of the early actinide mononitrides and dinitrides
}

\author{
K. O. Obodo ${ }^{1}$ \\ E-mail: Kingsley.Obodo@up.ac.za \\ ${ }^{1}$ Physics Department, University of Pretoria, Pretoria 0002, South Africa $;{ }^{2}$ National Institute \\ for Theoretical Physics,Johannesburg, 2000, South Africa
}

\section{N. Chetty ${ }^{1,2}$}

E-mail: Nithaya.Chetty@up.ac.za

${ }^{1}$ Physics Department, University of Pretoria, Pretoria 0002, South Africa; ${ }^{2}$ National Institute for Theoretical Physics,Johannesburg, 2000, South Africa

\begin{abstract}
We present a detailed comparative study of the electronic and mechanical properties of the early actinide mononitrides and dinitrides within the framework of the Perdew-Burke-Ernzerhof generalized gradient approximation (GGA [PBE]) and GGA $+U$ implementations of density functional theory with the inclusion of spin-orbit coupling. The dependence of selected observables of these materials on the effective $U$-parameter is investigated in detail. The properties include the lattice constant, bulk modulus, charge density distribution, hybridization of the atomic orbitals, energy of formation and the lattice dynamics. The inclusion of the Hubbard $U$ parameter results in a proper description of the $5 f$ electrons, and is subsequently used in the determination of the structural and electronic properties of these compounds. The mononitrides and dinitrides of the early actinides are metallic except for $\mathrm{UN}_{2}$, which is a semiconductor. These actinide nitrides are non-magnetic with the exception of $\mathrm{UN}, \mathrm{NpN}, \mathrm{PuN}, \mathrm{NpN}_{2}$ and $\mathrm{PuN}_{2}$ that are magnetic systems with orbital-dependent magnetic moments oriented in the z-axis. We observed that $\mathrm{ThN}_{2}$ is elastically unstable to isotropic pressure. We discovered that $\mathrm{UN}_{2}$ is thermodynamically unstable, but may be stabilized by $\mathrm{N}$ vacancy formation.
\end{abstract}

Submitted to: J. Phys.: Condens. Matter

PACS numbers: 62.20.D-, 63.20.D-, 71.15.Mb, 71.20.-b, 71.27.+a 


\section{Introduction}

The investigation of actinide-based materials is an area of active research. $[1,2,3,4,5,6,7$, $8,9,10,11,12]$ The effects of 5 -electrons in the actinide nitrides are of significant interest to both theorists and experimentalists, $[13,14,15]$ because exotic properties often arise from the strong electron-electron interactions paving the way for interesting physics and novel applications. The actinide metals exhibit increased $5 f$ electronic localization with increasing atomic number which has a direct bearing on the orbital overlap and electronic band structure of these systems.

Actinide nitrides are set to become a major component in large-scale energy generation devices in nuclear power stations as an alternative to the current fuel sources that are being used. $[16,17]$ The Generation-IV nuclear reactors (fast neutron fission reactors) are developed to use the actinide nitrides as the primary fuel source. [18] Actinide nitrides release a larger amount of fissile products (neutrons) compared to its consumption, and the long-lived fission products have a higher probability to transmute leading to higher usable energy densities.

Based on the importance of the actinide nitrides from both a scientific and technological point of view, the understanding of the electronic and structural properties is important to harness, control, and effectively use these materials. The presence of $5 f$ electrons poses a considerable challenge in the theoretical description of the actinide compounds. This is mainly due to the improper account of the electron-electron interactions by the local spin density approximation (LSDA) and the generalized gradient approximation (GGA) approaches implemented in density functional theory (DFT). The DFT $+U[19,20,21]$ $(\mathrm{LDA}+U$ and GGA $+U)$ approach has been shown to overcome some of the shortcomings and shows a lot of promise in the studies of the $5 f$ electron systems.

In this work, the actinide nitrides of interest are the early actinide mononitrides $(\mathrm{AcN})$ and dinitrides $\left(\mathrm{AcN}_{2}\right)$ where $(\mathrm{Ac}=\mathrm{Th}, \mathrm{Pa}, \mathrm{Np}, \mathrm{U}$ and $\mathrm{Pu})$. At low temperatures, the $\mathrm{AcN}$ crystallizes in the $\mathrm{NaCl}$ crystal structure, while the $\mathrm{AcN}_{2}$ mainly exists in the fluorite structure. There also exist other actinide compounds that crystallize in the $\mathrm{NaCl}$ crystal structure such as the mono-carbides, mono-pnictides, mono-chalcogenides, etc.

To the best of our knowledge, a detailed analysis of these systems within the DFT $+U$ approach is lacking. [22, 23, 10,24] Petit et al. [10] investigated some actinide mono-carbides and mononitrides using the self-interaction corrected (SIC) local spin-density approximation. These authors observed ionic character in the mononitrides, increased localization in PuN, and an itinerant picture for UN. Silva et al., [5] recently synthesized NpN and confirmed the existence of other phases of neptunium nitrides using both experimental and computational techniques. These authors showed the importance of the Hubbard $U$-parameter (accounting for onsite interactions) along with relativistic effects to accurately describe these compounds.

Atta-Fynn et al. [22] treated all the nitrides on equal footing at different magnetic configurations with and without spin orbit coupling (SOC). They showed that the type-I antiferromagnetic (AFM) configuration gives similar results to the type-II AFM configuration and results in a more energetically favorable configuration with the inclusion of SOC.

In our work, we have therefore considered the type-I AFM structure within the projector augmented wave (PAW) method to analyze the mononitride systems. We compare our results with available experimental data as well as with other theoretical results. All calculations are based on density functional theory with and without the inclusion of the Hubbard $U$ parameter $(\mathrm{DFT}+U)$. We evaluate the heat of formation, the structural, electronic, elastic, and mechanical properties of the various actinide nitrides. Of interest is how well our zero temperature DFT $+U$ computations predict these properties. Previous studies proposed that $\mathrm{PaN}$ has a more covalent character compared to $\mathrm{UN}, \mathrm{NpN}, \mathrm{PuN}$ that are predominantly ionic. 
Therefore, by scrutinizing the valence charge density distributions, we examine the $\mathrm{AcN}$ and $\mathrm{AcN}_{2}$ compounds to give an account of the bonding character of these systems. The Hubbard $U$-parameter is shown to be important in the prediction of the structural properties of the actinide nitrides. The lattice dynamics of these systems gives an indication of the mechanical stability.

The rest of this paper is organized as follows: in Section 2, we present a description of the computational methodology. The calculated ground state properties of $\mathrm{AcN}$ and $\mathrm{AcN}_{2}$ compounds, and the effect of the Hubbard $U$-parameter are given in Section 3 and 4 respectively. Finally, we make our concluding remarks in Section 5.

\section{Computational Details}

The calculations are performed using density functional theory [25] as implemented in the VASP code [26]. The PAW approach of Blöchl in the implementation of Kresse and Joubert is used to describe the electron wave functions. [27] The semi-core electrons and the valence electrons of the actinides are represented by plane waves. The PBE [28] form for the GGA and the GGA $+U$ is used in the description of the exchange-correlation potential.

The unit cell parameters and atomic positions are optimized with an energy tolerance of $10^{-7} \mathrm{eV}$. The calculations are performed with an energy cut-off of $500 \mathrm{eV}$ and $550 \mathrm{eV}$ for AcN and $\mathrm{AcN}_{2}$ respectively. The Brillouin zone (BZ) integrations are performed using a spacing of $0.2 \AA^{-1}$ for the Monkhorst-Pack [29] grid. Fractional electron occupancies and integration of the bands at the Fermi level are determined using the Methfessel-Paxton smearing method with a smearing parameter of $0.2 \mathrm{eV}$. The $\mathrm{AcN}$ and $\mathrm{AcN}_{2}$ with $\mathrm{SOC}$ (spin-orbit coupling) calculations are performed within the constraints of symmetry. The lifting of the symmetry constraints lead to no significant change in the total energy and electronic properties, with an exception of PuN and $\mathrm{UN}_{2}$ were the calculation is performed with the symmetry constraints lifted [3]. The total energy, electronic band structure and density of states (DOS) were calculated using the tetrahedron integration method with Blöchl corrections.

We use the Dudarev et al. [30] simplified rotationally-invariant form for the Hubbard correction to account for the on-site Coulomb interactions. This approach combines the Hubbard $U$ and Hund's $J$ to give the $U_{\text {eff }}=U-J$. The rotational invariance is preserved within this formalism because the $U$ parameter depends on the trace of the occupation matrices and their products. The generic expression for the energy functional in the DFT $+U$ formalism is represented by

$$
E_{D F T+U}=E_{D F T}+E_{H u b}-E_{d c},
$$

where $E_{D F T}$ is the DFT (LDA or GGA) contribution to the total energy and $E_{H u b}$ is the electron-electron interaction from the Hubbard term. $E_{d c}$ is a double counting term, introduced because part of the electron-electron interaction is taken into account in $E_{D F T}$. The last two terms depend on the occupation matrix of the correlated orbitals of the system under consideration. In this study, the calculated Hubbard $U_{\text {eff }}$ parameter (henceforth referred to as the Hubbard $U$ ) is optimized with respect to the experimentally determined lattice constant $[31,32,33,34,35,3]$ for each of the actinide nitrides that we considered. The optimized $U$ parameter is used to determine the structural, electronic, elastic and mechanical properties of the compounds. This results in a better qualitative and quantitative description of the various properties.

Relativistic effects are important for the actinides. This leads to lower calculated ground state total energies compared to non-relativistic calculations. In this study, we present results 
using the GGA and the GGA $+U$ forms for the exchange correlation potential together with spin-polarization (SP) and spin orbit coupling (SOC).

A method using the least squares fit, [36] as implemented in the MedeA-MT module, was used to obtain the elastic constants. The elastic properties are calculated from the Hill values, which are a geometric mean of the Voigt and Reuss values. The eigenvalues of the stiffness matrix give an indication of the mechanical stability of the systems under consideration. The Hill values [37] were used in the estimation of longitudinal, shear and mean sound velocities and the Debye temperatures. [38]

The energy of formation for each structure was calculated by taking the difference between the total energy of the actinide compound and the energies of its constituents in their corresponding bulk elemental forms:

$$
\Delta E_{f}=E_{\left(A c N_{n}\right)}^{t o t}-N_{A c} E_{(A c)}^{b u l k}-N_{N} E_{(N)}^{b u l k},
$$

where $n$ is either 1 or 2 for the mononitride and dinitride, respectively, and $N_{A c}$ and $N_{N}$ are the number of Ac atoms and $\mathrm{N}$ atoms in the calculational unit cell of the compound, respectively.

$E_{(A c)}^{b u l k}$ is the energy per Ac atom in its ground state structure, and $E_{(A c)}^{b u l k}$ is the energy per N atom in the nitrogen molecule.

The phonon frequencies are calculated using the direct approach of lattice dynamics based on linear response theory, as implemented in the MedeA-PHONON package. [39] This exploits the fact that within the harmonic regime, there is a linear relationship between the induced forces on the atoms and the displacement of the atoms from their equilibrium positions. We discovered that using GGA $+U$ only has a marginal effect on the quantitative description of the phonon frequencies. Therefore, we have only presented results for the phonon frequencies within the GGA approximation.

We applied our methods to the study of the ferromagnetic (FM) and the antiferromagnetic (AFM) configurations for the actinide mononitrides and dinitrides. The non-magnetic (NM) systems consistently resulted in higher energies of formation for all the structures considered. Therefore NM results are not presented in this study.

\section{AcN}

The AcN crystal structures are presented in Fig. 1. The ferromagnetic phase is the cubic $\mathrm{NaCl}$ structure with the $\mathrm{Fm}-3 \mathrm{~m}$ space group, and the antiferromagnetic phase is cubic $\mathrm{NaCl}$ with the $P m 3 m$ space group.

\subsection{Energy of Formation}

In Table 1, the calculated equilibrium lattice constant, energy of formation, magnetic moment, bulk and shear moduli, and Debye temperature are presented. The energy of formation for all the AcN investigated, calculated using equation 2, is negative. This implies that the formation of these structures is energetically favorable.

Also, in Table 1 we observe that $\mathrm{ThN}$ and $\mathrm{PaN}$ evolve to their non-magnetic ground states with the AFM structure being slightly more energetically favorable than the FM structure. $\mathrm{UN}$ to PuN give various degrees of magnetization due to the $5 f$ orbitals, with the magnetic orientation along the z-axis in both the AFM and FM ordering. This is attributed to strong electron-electron correlations of the $5 f$ electrons at each atomic site resulting in net local magnetic moments. We observe that the degree of magnetization is over-estimated using the PAW $(\mathrm{GGA}+U)$ approach compared to all-electron (AE) calculations and experimental data. 
Table 1: The Hubbard $U(\mathrm{eV})$, optimize lattice parameters $a$ (in $\AA$ ), calculated energy of formation $\Delta E_{f}(\mathrm{eV})$, total magnetic moment $\mu_{B}$ (Bohr magnetons), bulk and shear moduli $(\mathrm{GPa})$, and the Debye temperature $\theta_{D}(\mathrm{~K})$ using GGA $+U$ within the SOC scheme. Where $\mathrm{AE}$ results is from Ref [22] and the PAW results is our data.

\begin{tabular}{|c|c|c|c|c|c|c|c|c|c|}
\hline Compound & Method & Theory & $U$ & $a_{0}$ & $\Delta E_{f}$ & $\mu_{B}$ & bulk & shear & $\theta_{D}$ \\
\hline \multirow{4}{*}{ ThN } & \multirow{2}{*}{ PAW } & $\mathrm{AFM}+\mathrm{SOC}$ & 0 & 5.172 & -3.048 & 0 & 178 & 83 & 340.7 \\
\hline & & $\mathrm{FM}+\mathrm{SOC}$ & 0 & 5.171 & -3.040 & 0 & 178 & 82 & 338.4 \\
\hline & \multirow{2}{*}{$\mathrm{AE}$} & $\mathrm{AFM}+\mathrm{SOC}$ & 0 & 5.175 & - & 0 & 170 & - & - \\
\hline & & $\mathrm{FM}+\mathrm{SOC}$ & 0 & 5.175 & - & 0 & 162 & - & - \\
\hline \multirow{6}{*}{$\mathrm{PaN}$} & \multirow{4}{*}{ PAW } & $\mathrm{AFM}+\mathrm{SOC}$ & 0 & 4.952 & -3.225 & 0 & 219 & 95 & 358 \\
\hline & & $\mathrm{FM}+\mathrm{SOC}$ & 0 & 4.951 & -3.228 & 0 & 220 & 88 & 345.8 \\
\hline & & $\mathrm{AFM}+\mathrm{SOC}$ & 1 & 4.965 & -2.636 & 0 & 223 & 102 & 371.1 \\
\hline & & $\mathrm{FM}+\mathrm{SOC}$ & 1 & 4.965 & -2.639 & 0 & 224 & 94 & 357.3 \\
\hline & \multirow{2}{*}{$\mathrm{AE}$} & $\mathrm{AFM}+\mathrm{SOC}$ & 0 & 4.947 & - & 0 & 200 & - & - \\
\hline & & $\mathrm{FM}+\mathrm{SOC}$ & 0 & 4.953 & - & 0 & 210 & - & - \\
\hline \multirow{6}{*}{ UN } & \multirow{4}{*}{ PAW } & $\mathrm{AFM}+\mathrm{SOC}$ & 0 & 4.871 & -2.643 & 0 & 201 & 72 & 305.2 \\
\hline & & $\mathrm{FM}+\mathrm{SOC}$ & 0 & 4.872 & -2.663 & 1.0976 & 206 & 85 & 316.2 \\
\hline & & $\mathrm{AFM}+\mathrm{SOC}$ & 1 & 4.903 & -1.685 & 0 & 196 & 63 & 288.5 \\
\hline & & $\mathrm{FM}+\mathrm{SOC}$ & 1 & 4.889 & -1.713 & 1.3713 & 198 & 52 & 262.9 \\
\hline & \multirow{2}{*}{$\mathrm{AE}$} & $\mathrm{AFM}+\mathrm{SOC}$ & 0 & 4.873 & - & 0 & 221 & - & - \\
\hline & & $\mathrm{FM}+\mathrm{SOC}$ & 0 & 4.873 & - & 0.96 & 219 & - & - \\
\hline \multirow{6}{*}{$\mathrm{NpN}$} & \multirow{4}{*}{ PAW } & $\mathrm{AFM}+\mathrm{SOC}$ & 0 & 4.863 & -2.299 & 0 & 178 & 78 & 317.3 \\
\hline & & $\mathrm{FM}+\mathrm{SOC}$ & 0 & 4.859 & -2.341 & 2.4658 & 178 & 79 & 320.4 \\
\hline & & $\mathrm{AFM}+\mathrm{SOC}$ & 1 & 4.897 & -1.268 & 0 & 167 & 71 & 303.5 \\
\hline & & $\mathrm{FM}+\mathrm{SOC}$ & 1 & 4.909 & -1.299 & 3.0399 & 145 & 49 & 253.1 \\
\hline & \multirow{2}{*}{$\mathrm{AE}$} & $\mathrm{AFM}+\mathrm{SOC}$ & 0 & 4.863 & - & 0 & 195 & - & - \\
\hline & & $\mathrm{FM}+\mathrm{SOC}$ & 0 & 4.863 & - & 2.45 & 183 & - & - \\
\hline \multirow{4}{*}{ PuN } & \multirow{2}{*}{ PAW } & $\mathrm{AFM}+\mathrm{SOC}$ & 0 & 4.907 & -6.580 & 0 & 159 & 86 & 328.1 \\
\hline & & $\mathrm{FM}+\mathrm{SOC}$ & 0 & 4.916 & -6.404 & 4.2438 & 147 & 89 & 335 \\
\hline & \multirow{2}{*}{$\mathrm{AE}$} & $\mathrm{AFM}+\mathrm{SOC}$ & 0 & 4.9531 & - & 0 & 141 & - & - \\
\hline & & $\mathrm{FM}+\mathrm{SOC}$ & 0 & 4.9740 & - & 4.26 & 145 & - & - \\
\hline
\end{tabular}




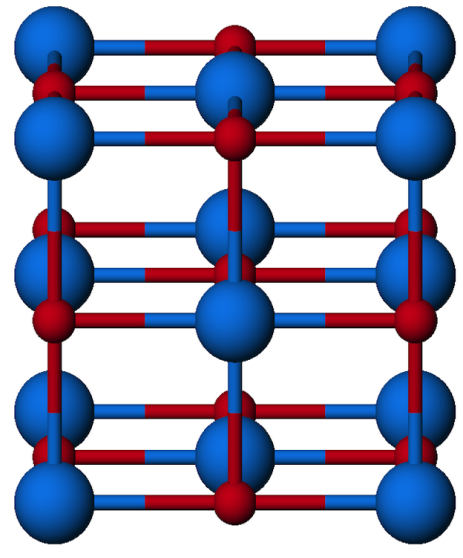

(a) $\mathrm{AcN}-\mathrm{fm}$

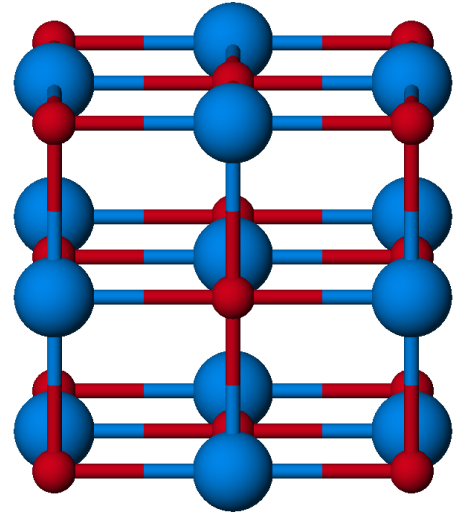

(b) $\mathrm{AcN}-\mathrm{afm}$

Figure 1: Crystal Structure for AcN compounds in the FM and AFM phase

For the UN to PuN compounds, the total energy with PAW (with and without the Hubbard $U$ ) results in the FM structure having the lowest energy of formation.

\subsection{Structural Properties}

We present the lattice constants calculated using the PAW method (GGA with and without the inclusion of Hubbard $U$-parameter) in Table 1. We find a favorable comparison with the $\mathrm{AE}$ [22] results for the various AcN compounds considered. For ThN, we obtain reasonable results without the inclusion of the Hubbard $U$ parameter. This is understandable since Th lacks $5 f$ electrons. Also for PuN, the lattice constant is not affected much by the inclusion of the $U$. This is understood in terms of its relative position in the actinide series.

The inclusion of the Hubbard $U$ parameter of $1 \mathrm{eV}$ in $\mathrm{UN}$ and $\mathrm{NpN}$ results in lattice constants that compare very well with experiment. The experimentally determined lattice constant $[40,41]$ and the calculated result of our lowest energy structure in parenthesis for ThN, UN, NpN and PuN are 5.154 ̊ (5.172 ̊), 4.889 ̊ (4.889 ̊), $4.894 \AA$ (4.909 ̊) and $4.905 \AA$ (4.907 ̊̊) respectively.

\subsection{Elastic Properties}

The calculated bulk modulus, shear modulus and Debye temperature for the $\mathrm{AcN}$ compounds are presented in Table 1. They are all positive, which indicates that the compounds are all elastically stable. The inclusion of the $U$ parameter leads to an overall better agreement of the calculated elastic properties with other available theoretical and experimental data [41, 42, $22,8]$. For instance, the experimentally determined bulk modulus and the calculated result of our lowest energy structure in parenthesis for ThN and UN are $175 \mathrm{GPa}$ [43] (178 GPa) and $193 \mathrm{GPa}[41]$ (198 GPa) respectively.

$\mathrm{PaN}$ has the highest Debye temperature $\theta_{D}$ of $358 \mathrm{~K}$ of the systems that we considered. This is associated with complex covalent bonding behavior in this system that will be further explored in our discussion on the valence charge density. PaN, followed by $\mathrm{UN}$, have the 
highest calculated bulk modulus that also points to the inherent covalent bonding in these systems.

\subsection{Density of States}

The partial density of states (PDOS) for the $5 f$ and $6 d$ states for the actinides and the $2 p$ states of the nitrogen are presented for the ground state AcN compounds. All the compounds studied within GGA and GGA $+U$ are found to be metallic. There is a systematic increase in the $5 f$ electron density of states at the Fermi level with increasing atomic number, and there is a contribution from the $\mathrm{N} 2 \mathrm{p}$ states below the Fermi level.

As shown in Fig. 2 for the AFM structure of ThN, the metallicity of ThN is determined mainly by the hybridization of the $5 f$ and $6 d$ states from the actinide with a very minute contribution from $2 p$ states of the nitrogen at the Fermi level.

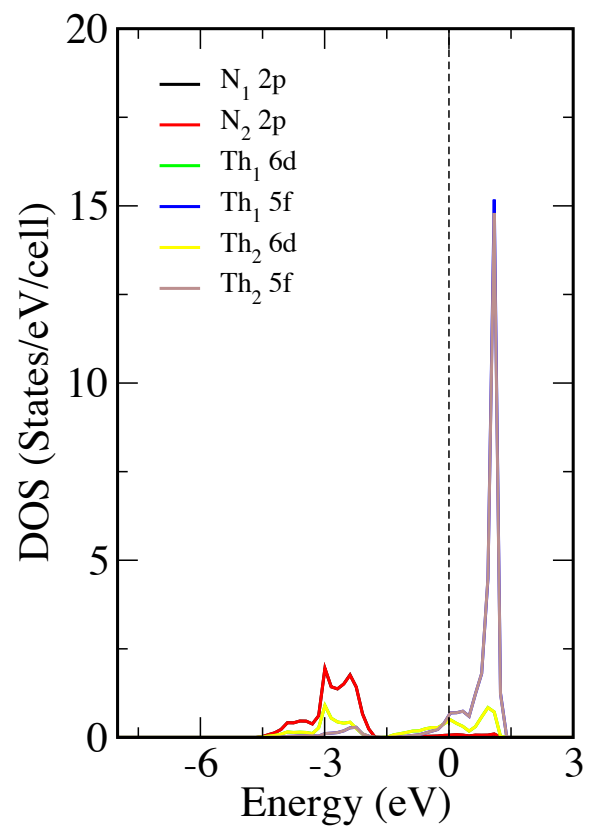

Figure 2: (Color online) Partial density of states for ThN in the AFM structure. The Fermi level is the vertical line through $\mathrm{E}=0$

From PaN to PuN, we have a higher $5 f$ electron contribution at the Fermi level compared to the other orbitals in both the GGA and GGA $+U$ descriptions shown in figures 3 . This is attributed to increased localization of the $5 f$ electrons in the actinides. However, other experimental and theoretical studies $[14,15,7]$ of $\mathrm{UN}$ have confirmed that the $\mathrm{U} 5 f$ states have some itinerant character that is attributed to the presence of $U 5 f$ states at the Fermi-level therefore preserving some band character. [15]

In Fig 4, we present the partial DOS for PuN in the AFM structure. There is a significant difference in the peaks of the two actinide atoms of the $5 f$ states (minority and majority spin) compared to the AFM PDOS of ThN that has no difference in the peaks. This is an indication 


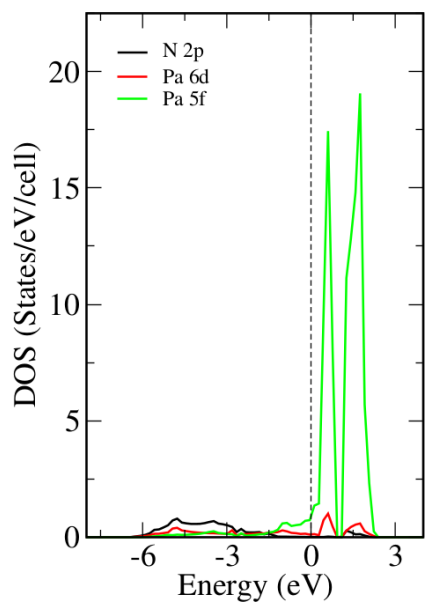

(a) $\mathrm{PaN} \mathrm{U}=0$

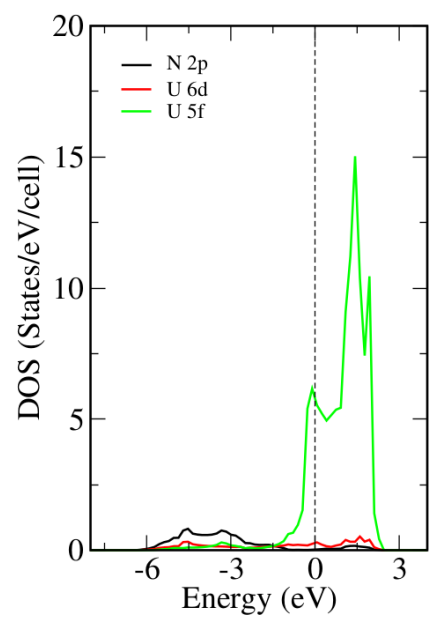

(d) $\mathrm{UN} \mathrm{U}=1$

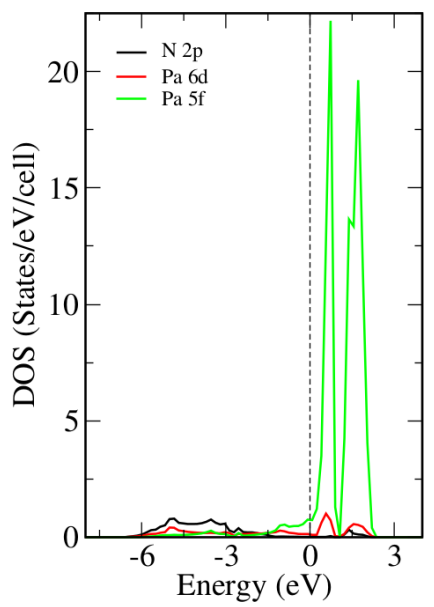

(b) $\mathrm{PaN} \mathrm{U}=1$

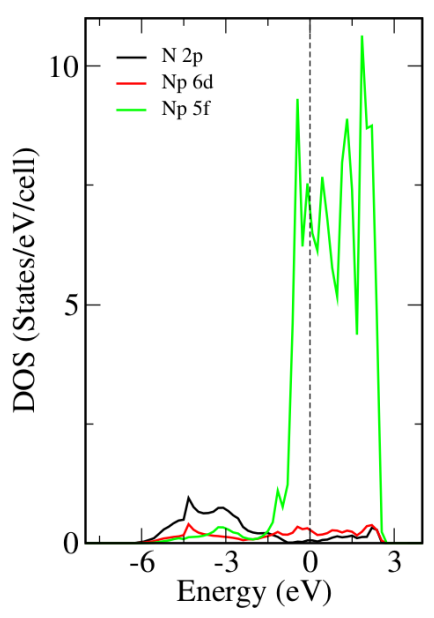

(e) $\mathrm{NpN} \mathrm{U}=0$

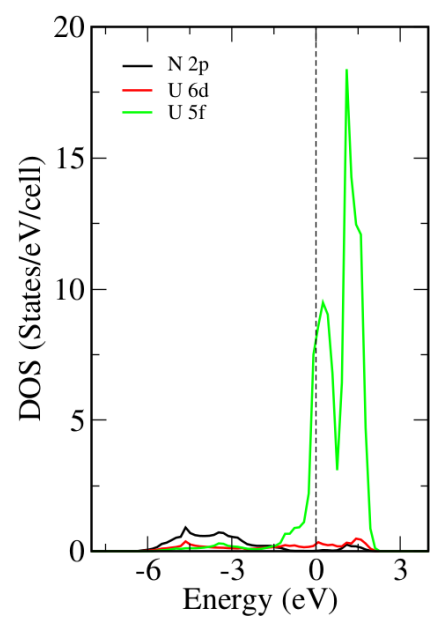

(c) $\mathrm{UN} \mathrm{U}=0$

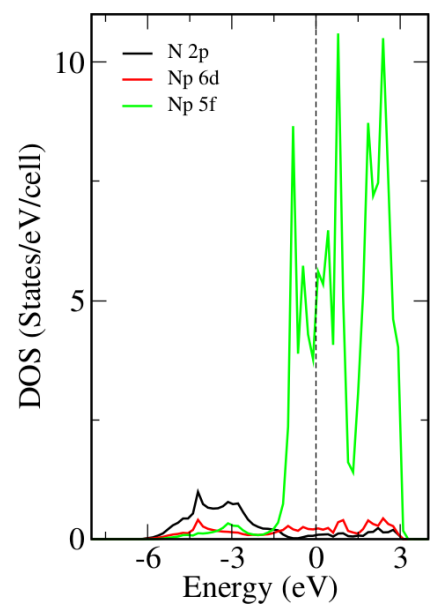

(f) $\mathrm{NpN} \mathrm{U}=1$

Figure 3: (Color online) Partial density of states for PaN, $\mathrm{UN}$ and $\mathrm{NpN}$ in the FM structure. The Fermi level is the vertical line through $\mathrm{E}=0$

of AF magnetic ordering in this system. Similar features are observed in the AFM structure of UN to PuN.

\subsection{Valence charge densities}

In Fig. 5, we show the valence charge density plots for the compounds that we investigated. This gives us an indication of the character of the bonding. The charge density distribution is computed in the (001) plane for the AFM configuration. The scale in Fig. 5f is such that blue is devoid of electrons and red indicates a high concentration of electrons. The charge densities plotted are for the valence states summed over both spin orientations. 


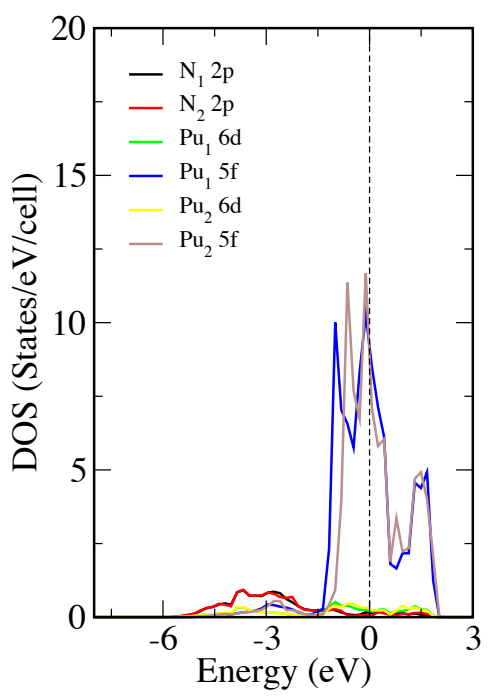

Figure 4: (Color online) Partial density of states for PuN in the AFM structure. The Fermi level is the vertical line through $\mathrm{E}=0$

In general, we observe near-spherical charge density distributions around the actinide and nitrogen atoms with significant charge depletion between the atoms, which is characteristic of the rock-salt structure with predominant ionic bonding. Between the actinide and nitrogen atoms there is a subtle charge density bridge, attributed to the slight covalent character in the actinide nitrides. There is charge transfer from the actinide metals to the non-metallic nitrogen atoms. As the atomic number increases (Th to $\mathrm{Pu}$ ) resulting in higher valence localized $5 f$ electrons, there is a noticeable increase in the charge accumulation at the actinide site.

\subsection{Phonon Studies}

In Fig. 6, no negative phonon modes for the various AcN structures are observed in the phonon dispersion plot. Therefore, these compounds are dynamically stable. The low-lying frequency modes are governed by the dynamics of the heavy Ac atoms, and the higher frequencies by the lighter $\mathrm{N}$ atoms. There is a significant difference in the frequency of $\mathrm{N}$ atoms compared to the Ac atoms due to the large mass differences. This suggests that instability in the system can arise from a small perturbation of the crystal structure.

PuN has the lowest acoustic velocity corresponding to the lowest bulk modulus and overall strength of all the AcN systems that we studied. There are "flatish" sections of the optical branches in all systems that suggest low group velocities for these modes involving $\mathrm{N}$.

\section{4. $\mathrm{AcN}_{2}$}

In Fig. 7, we present the $\mathrm{AcN}_{2}$ crystal structures. The ferromagnetic (FM) phase is the cubic fluorite structure with space group $F m-3 m$, while the antiferromagnetic phase (AFM) is a double tetragonal unit cell with space group $P 4 / \mathrm{mmm}$, and the magnetic ordering is in the z-axis. 


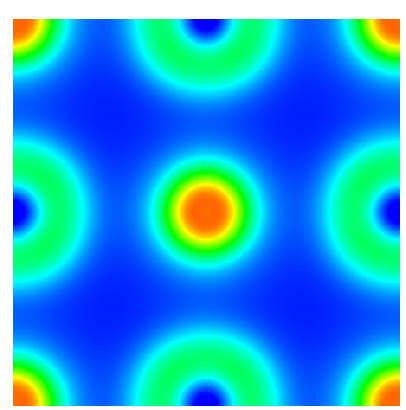

(a) $\mathrm{ThN}$

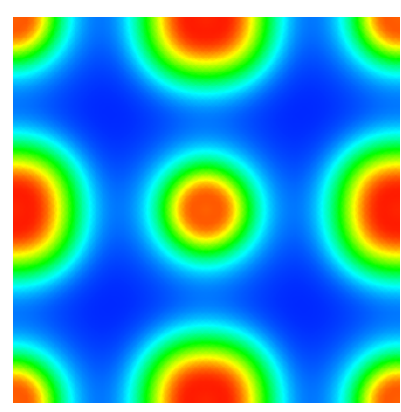

(d) $\mathrm{NpN}$

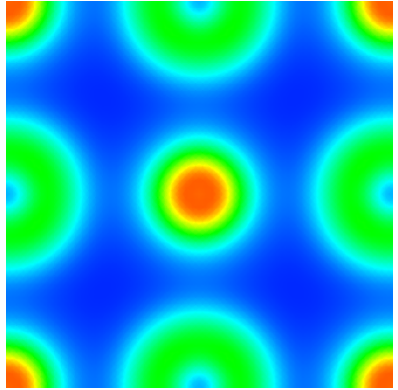

(b) $\mathrm{PaN}$

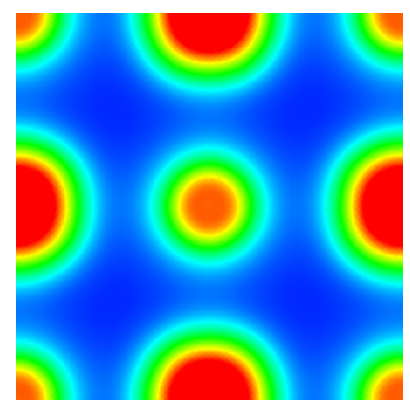

(e) $\mathrm{PuN}$

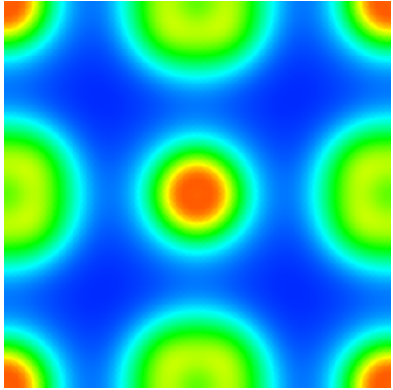

(c) UN

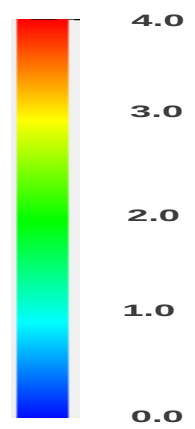

(f) Scale (in e/ $/ \AA^{3}$ )

Figure 5: Valence charge density in the (001) direction of the AFM structure for AcN crystalline structures, where the charge distribution at the center and corner of the slice is from $\mathrm{N}$ atom, and that at face of the slice is from the Ac atom. Color scale: deep blue represents low valence charge while red represents a high concentration of valence charge.

\subsection{Energy of Formation}

In Table 2, the calculated equilibrium lattice constant, energy of formation, magnetic moment, bulk and shear moduli, and Debye temperature are presented. The calculated energy of formation for the $\mathrm{AcN}_{2}$, using equation 2, is negative, which implies that the structures are energetically favorable. The difference in the energy of formation of the $\mathrm{AcN}_{2}$ within the GGA approach using the AFM and FM structures is about one-hundredth of an $\mathrm{eV}$. The introduction of the Hubbard $U$ parameter results in a difference of about one-tenth of an $\mathrm{eV}$ in the energy of formation between the AFM and the FM structures. We conclude that the AFM structures are slightly more energetically favorable with the exception of $\mathrm{ThN}_{2}$ for which the FM is more energetically favorable.

$\mathrm{ThN}_{2}$ to $\mathrm{UN}_{2}$ compounds evolve to the non-magnetic ground state irrespective of the starting magnetic configuration. We observe that the magnetic orientation has little effect on the structural, elastic and mechanical properties. For $\mathrm{NpN}_{2}$ and $\mathrm{PuN}_{2}$, the AFM structure results in zero net magnetization. But, the FM structure for $\mathrm{NpN}_{2}$ gives a net magnetic 


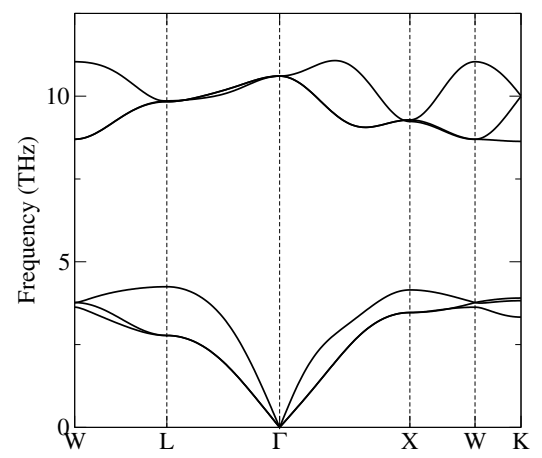

(a) $\mathrm{ThN}$

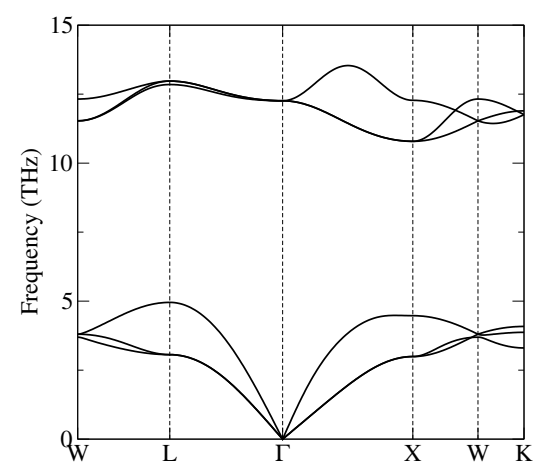

(c) UN

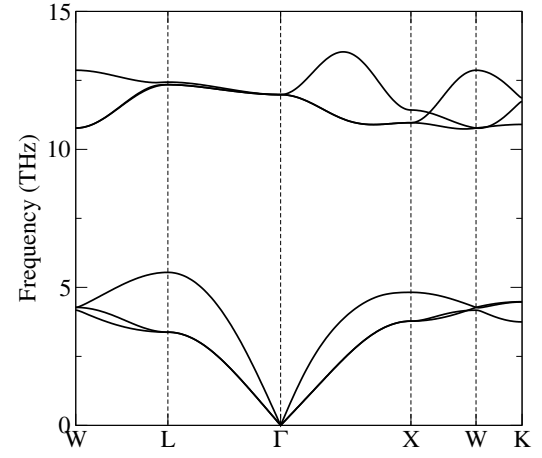

(b) $\mathrm{PaN}$

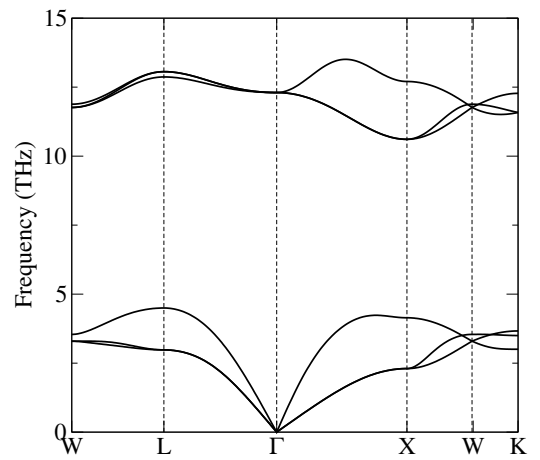

(d) $\mathrm{NpN}$

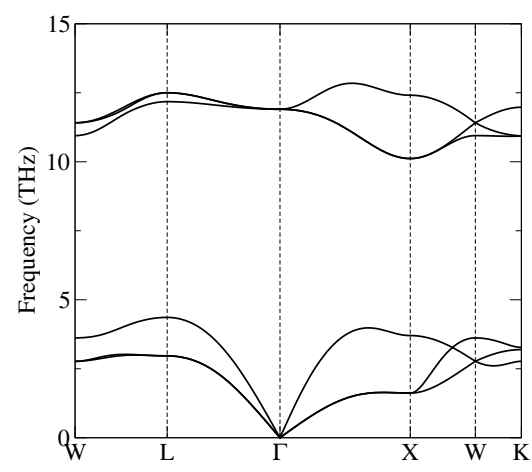

(e) PuN

Figure 6: Phonon dispersion for AcN crystalline structures

moment of $0.19 \mu_{B}$ and $0.95 \mu_{B}$ for GGA and GGA $+U$ respectively. The FM structure for $\mathrm{PuN}_{2}$ gives a net magnetic moment of $1.74 \mu_{B}$ for GGA and $2.04 \mu_{B}$ for GGA $+U$. The magnetic orientation in these compounds is along the $\mathrm{z}$-axis.

Comparing the energy of formation for the actinide mononitrides and dinitrides, we observe that the dinitrides are more energetically stable with the exception of $\mathrm{ThN}_{2}$ that is later shown to be elastically unstable. $\mathrm{PuN}_{2}$ has a slightly lower energy of formation than 


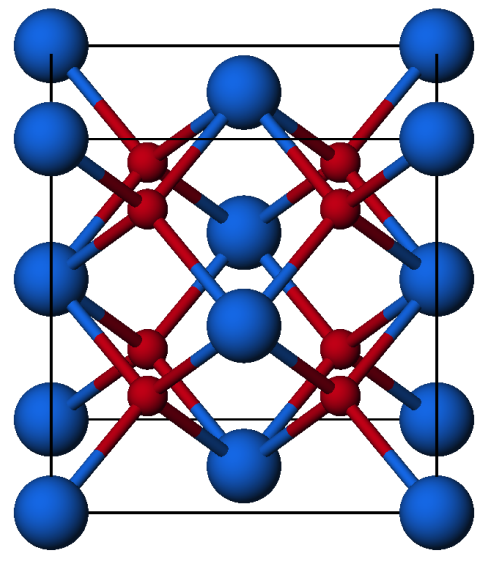

(a) $\mathrm{AcN}_{2}-\mathrm{fm}$

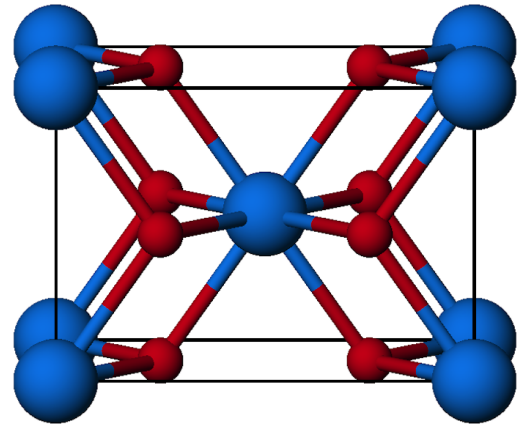

(b) $\mathrm{AcN}_{2}$-afm

Figure 7: Crystal Structure for $\mathrm{AcN}_{2}$ compounds in the FM and AFM phase

PuN.

\subsection{Structural Properties}

In Table 2, we present the calculated equilibrium lattice constant, magnetic moment, bulk and shear moduli and Debye temperature. The lattice constant using the PAW approach obtained for the various $\mathrm{AcN}_{2}$ compounds considered is calculated using GGA and GGA $+U$. The experimentally determined lattice constant and the calculated result with the Hubbard $U$ parameter of our lowest energy structure in parenthesis for $\mathrm{UN}_{2}[40,44]$ and $\mathrm{NpN}_{2}[5]$ are $5.308 \AA(5.302 \AA)$ and $5.3236 \AA(5.32 \AA)$ respectively. To the best of our knowledge, no experimental data on the lattice parameters of $\mathrm{ThN}_{2}, \mathrm{PaN}_{2}$ and $\mathrm{PuN}_{2}$ is available.

\subsection{Elastic properties}

The $\mathrm{AcN}_{2}$ considered are all elastically stable except for $\mathrm{ThN}_{2}$, which is elastically unstable to shear stress due to the negative shear modulus. The Debye temperature therefore cannot be calculated for this system. This is consistent with experimental findings that suggest the existence of two stable alloys of thorium and nitrogen, namely $\mathrm{ThN}$ and $\mathrm{Th}_{3} \mathrm{~N}_{4}$. [45]

The bulk and shear moduli increases from $\mathrm{ThN}_{2}$ to $\mathrm{UN}_{2}$, then subsequently decreases to $\mathrm{PuN}_{2}$. The calculated bulk modulus, shear modulus and Debye temperature with and without the $U$ parameter for $\mathrm{PaN}_{2}$ and $\mathrm{UN}_{2}$ are consistent for the AFM and the FM phases as shown in Table 2. Also, for $\mathrm{NpN}_{2}$ without the $U$ parameter, the elastic properties are consistent for the AFM and FM phase. However, we discovered that the FM phase for both $\mathrm{NpN}_{2}$ and $\mathrm{PuN}_{2}$ are highly sensitive to structural optimization, which leads to significant structural distortion.

\subsection{Density of States}

The partial density of states (PDOS) for the $5 f$ and $6 d$ states for the actinide atom and the $2 p$ states of the nitrogen atom is presented for $\mathrm{AcN}_{2}$ in the ground state structures. We observe 
Table 2: The Hubbard $U$ (eV), optimize lattice parameters $a$ (in $\AA$ ), calculated energy of formation $\Delta E_{f}(\mathrm{eV})$, total magnetic moment (scalar norm) $\mu_{B}$ (Bohr magnetons), bulk and shear moduli $(\mathrm{GPa})$, and the Debye temperature $\theta_{D}(\mathrm{~K})$ using GGA $+U$ within the SOC scheme.

\begin{tabular}{rcccccccc}
\hline \hline Compound & Theory & $U$ & $a_{0}$ & $\Delta E_{f}$ & $\mu_{B}$ & bulk & shear & $\theta_{D}$ \\
\hline \multirow{2}{*}{ ThN2 } & AFM+SOC & 0 & 5.660 & -1.590 & 0.00 & 145.6 & -9.4 & - \\
& FM+SOC & 0 & 5.664 & -1.636 & 0.00 & 150.0 & -4.3 & - \\
& & & & & & & & \\
\multirow{5}{*}{ PaN2 } & AFM+SOC & 0 & 5.429 & -3.777 & 0.00 & 214.3 & 89.8 & 405.7 \\
& FM+SOC & 0 & 5.418 & -3.762 & 0.00 & 217.3 & 89.2 & 404.5 \\
& AFM+SOC & 2 & 5.460 & -2.551 & 0.00 & 216.9 & 87.1 & 401.1 \\
& FM+SOC & 2 & 5.451 & -2.532 & 0.00 & 217.3 & 81.5 & 388.5 \\
& & & & & & & & \\
& AFM+SOC & 0 & 5.275 & -4.094 & 0.00 & 249.8 & 98.1 & 413.3 \\
& FM+SOC & 0 & 5.271 & -4.072 & 0.00 & 250.0 & 98.5 & 414.1 \\
& AFM+SOC & 2 & 5.302 & -2.135 & 0.00 & 249.2 & 89.1 & 395.7 \\
& FM+SOC & 2 & 5.297 & -2.088 & 0.00 & 251.7 & 89.3 & 396.0 \\
& & & & & & & & \\
& AFM+SOC & 0 & 5.223 & -3.089 & 0.00 & 235.2 & 93.7 & 402.4 \\
& FM+SOC & 0 & 5.219 & -3.015 & 0.19 & 237.4 & 94.3 & 403.7 \\
PuN2 & AFM+SOC & 3 & 5.320 & 0.086 & 0.00 & 198.6 & 68.4 & 347.5 \\
& FM+SOC & 0 & 5.222 & -6.209 & 1.74 & 209.6 & 74.4 & 354.7 \\
& FM+SOC & 3 & 5.307 & 0.518 & 0.95 & 193.3 & 86.5 & 408.6 \\
& FM+SOC & 3 & 5.321 & -3.303 & 0.00 & 149.3 & 39.6 & 263.8 \\
& AFM+SOC & 0 & 5.351 & -2.854 & 2.04 & 163.2 & 68.1 & 342.6 \\
\hline \hline & & & & & & & &
\end{tabular}

that $\mathrm{AcN}_{2}$ within the GGA and GGA $+U$ are metallic with the exception of $\mathrm{UN}_{2}$ which has a band gap.

In Fig. 10, an insulating character is observed for $\mathrm{UN}_{2}$ with a GGA band gap of $0.56 \mathrm{eV}$ and an increase in the band gap to $0.64 \mathrm{eV}$ on the inclusion of the Hubbard $U$ of $2 \mathrm{eV}$. This is comparable to the band gap obtained by Weck et al. [23] of $0.94 \mathrm{eV}$ using the AE approach and by Lu et al. [8] of $0.75 \mathrm{eV}$ using a Hubbard $U$ of $4 \mathrm{eV}$. If we consider the FM structure, which is less energetically favorable, a GGA band gap of $0.73 \mathrm{eV}$ and a band gap of $0.83 \mathrm{eV}$ on inclusion of the Hubbard $U$ of $2 \mathrm{eV}$ (figure not presented) is obtained. The top of the valence band is determined mainly by the hybridization of the $\mathrm{U} 5 f$ and $\mathrm{N} 2 p$ states, while the electronic properties of $\mathrm{UN}_{2}$ is governed mainly by the $5 f$ states because they dominate the conduction band minimum and valence band maximum.

The metallicity of $\mathrm{ThN}_{2}$ is determined mainly by the $2 p$ states as shown in Fig. 8. There is significant hybridization of the $5 f$ and $6 d$ states for the thorium atom and the $2 p$ states of the nitrogen atom at the Fermi level.

While in $\mathrm{PaN}_{2}$ as shown in Fig. 9, there is approximately equal participation of the $5 f$ and $6 d$ states around the Fermi level compared to the other orbitals in both the GGA and GGA $+U$. The inclusion of the Hubbard $U$ of $2 \mathrm{eV}$ leads to an increase in the $5 f$ states in the conduction band but no noticeable change at the Fermi level. 


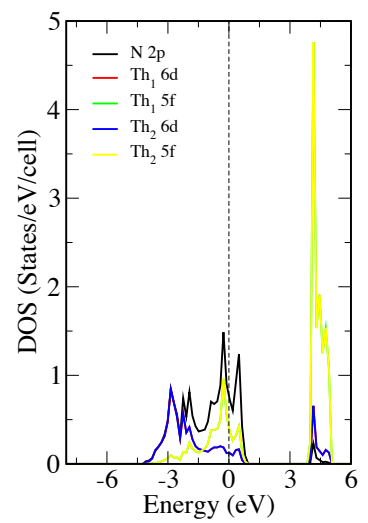

Figure 8: (Color online) Partial density of states for $\mathrm{ThN}_{2}$. The Fermi level is the vertical line through $\mathrm{E}=0$

The $\mathrm{NpN}_{2}$ and $\mathrm{PuN}_{2}$ compounds results in metallic behavior as presented in Fig. 11 and 12 with the Fermi level dominated by the $5 f$ states. There is weak hybridization of the actinide $5 f$ and $6 d$ states at the Fermi-level in $\mathrm{NpN}_{2}$ and a significant hybridization of the actinide $5 f$ and $6 d$ states with the nitrogen $2 p$ state in $\mathrm{PuN}_{2}$ compound.

\subsection{Valence charge densities}

In Fig. 13, we show the valence charge density plots for all the compounds considered to investigate the bonding character. The density distribution is computed in the (100) plane for the AFM configuration. We observe that the bonding character is not dependent on the magnetic configuration of the systems that we investigated. The scale in Fig. 13f is such that blue is devoid of electrons and red indicates a high concentration of electrons. The charge densities plotted are for the valence states summed over both spin orientations.

We observe significant distortion in the spherical charge distribution around the actinide compared to the nitrogen atoms. Between the actinide and nitrogen atom there is a subtle charge density bridge, attributed to the slight covalent character in the actinide nitrides. This charge density bridge along with the actinide charge density increases with increase in the atomic number of the actinide metal (Th to $\mathrm{Pu}$ ) compared to the rest of the interstitial region. This results in increased covalent bonding character of the actinide nitride with increase in the atomic number. However, the $\mathrm{AcN}_{2}$ studied are all predominantly ionic compounds.

\subsection{Phonon Studies}

The calculated phonon dispersion curves along the W-L- $-\mathrm{X}-\mathrm{W}-\mathrm{K}$ directions for $\mathrm{AcN}_{2}$ are presented in Fig. 14 within the GGA approach. Generally, the phonon frequencies for all the compounds considered are split roughly into two halves, where the lower frequencies are governed by the dynamics of the heavy Ac atoms and the higher frequencies by the dynamics of the $\mathrm{N}$ atoms.

The $\mathrm{AcN}_{2}$ are dynamically stable with no negative phonon modes in the dispersion plot with the important exception for $\mathrm{UN}_{2}$, which has a small fraction of imaginary phonon frequencies. This is due to the transverse modes of the nitrogen atoms. 


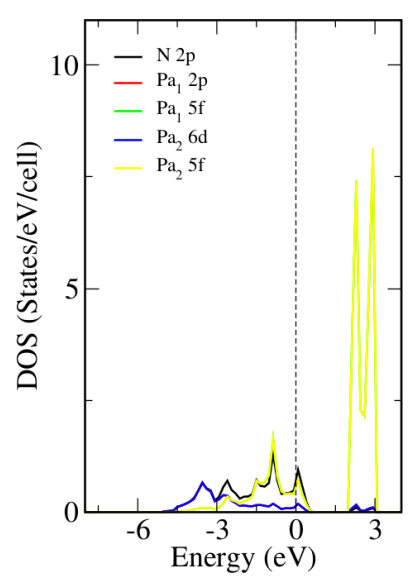

(a) $\mathrm{U}=0$

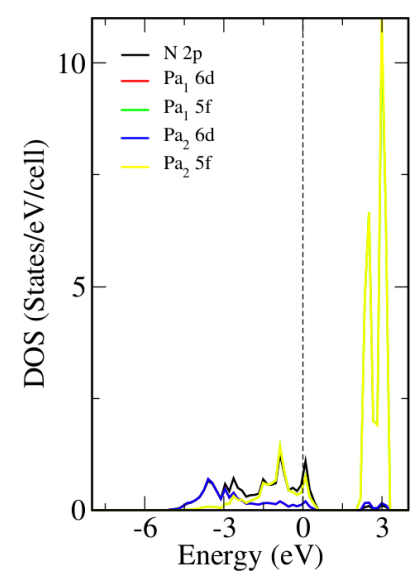

(b) $\mathrm{U}=2$

Figure 9: (Color online) Partial density of states for $\mathrm{PaN}_{2}$. The Fermi level is the vertical line through $\mathrm{E}=0$

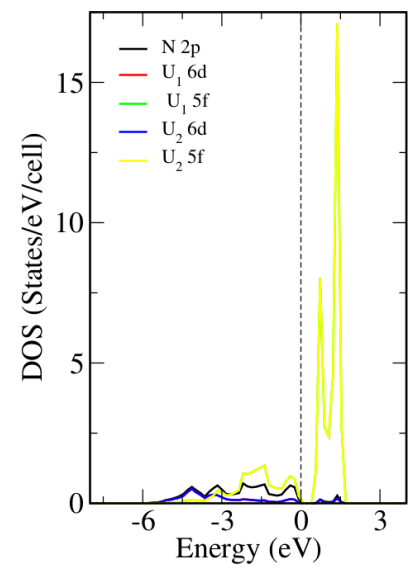

(a) $U=0$

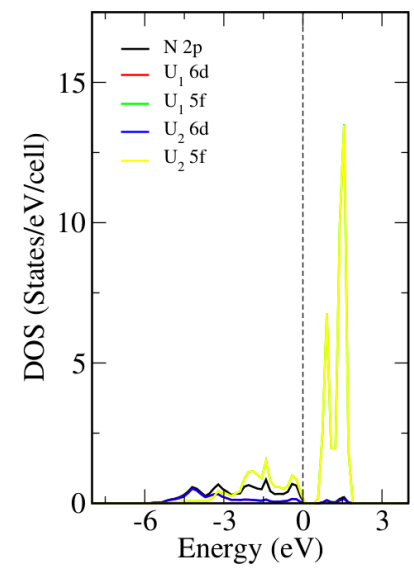

(b) $U=2$

Figure 10: (Color online) Partial density of states for $\mathrm{UN}_{2}$. The Fermi level is the vertical line through $\mathrm{E}=0$ 


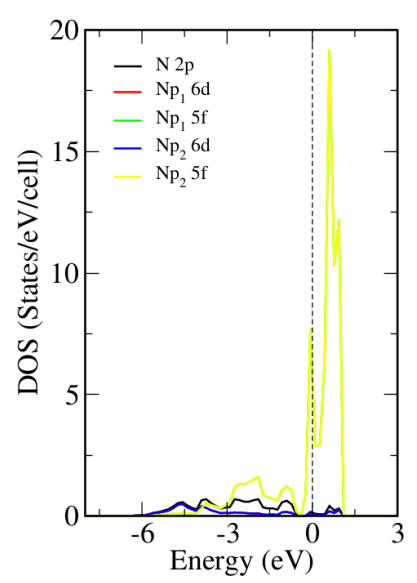

(a) $\mathrm{U}=0$

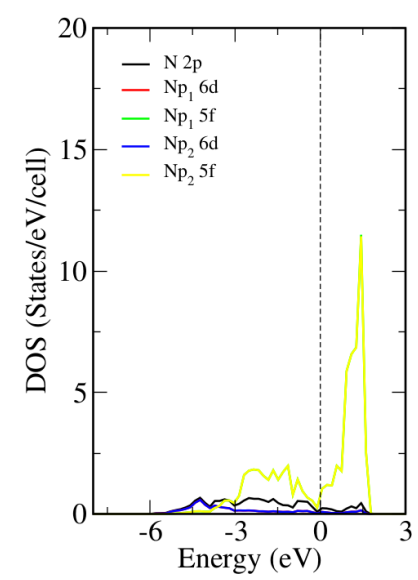

(b) $\mathrm{U}=3$

Figure 11: (Color online) Partial density of states for $\mathrm{NpN}_{2}$. The Fermi level is the vertical line through $\mathrm{E}=0$

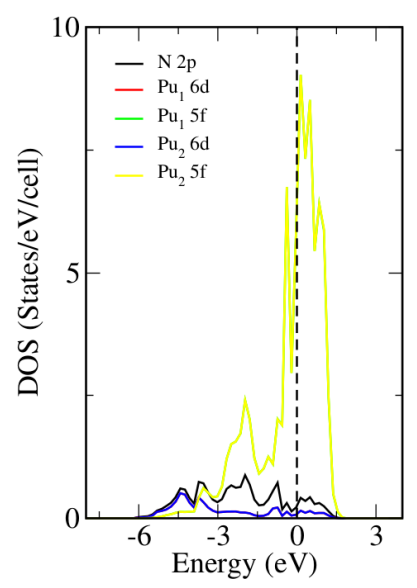

(a) $U=0$

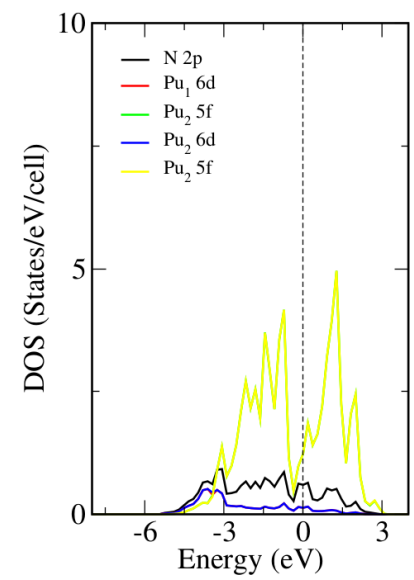

(b) $\mathrm{U}=3$

Figure 12: (Color online) Partial density of states for $\mathrm{PuN}_{2}$. The Fermi level is the vertical line through $\mathrm{E}=0$ 


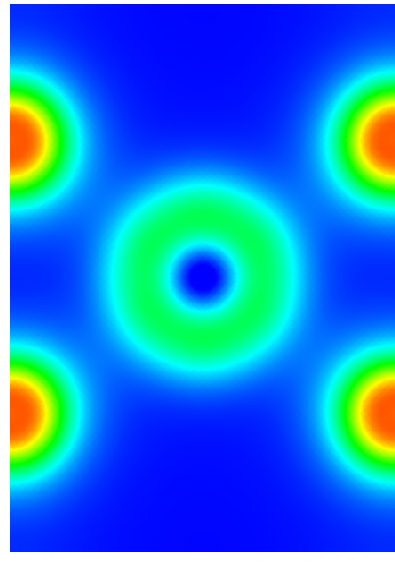

(a) $\mathrm{ThN}_{2}$

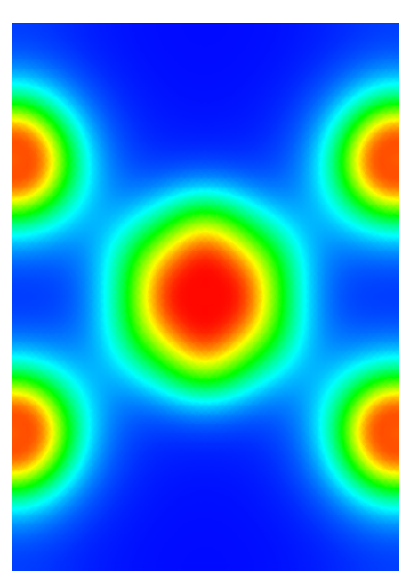

(d) $\mathrm{NpN}_{2}$

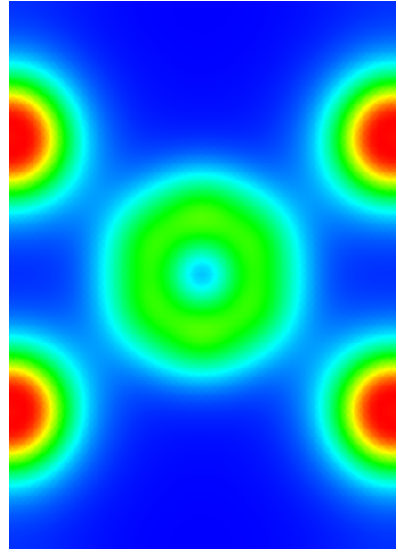

(b) $\mathrm{PaN}_{2}$

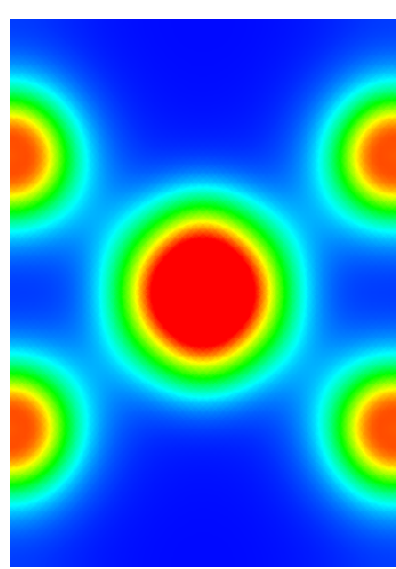

(e) $\mathrm{PuN}_{2}$

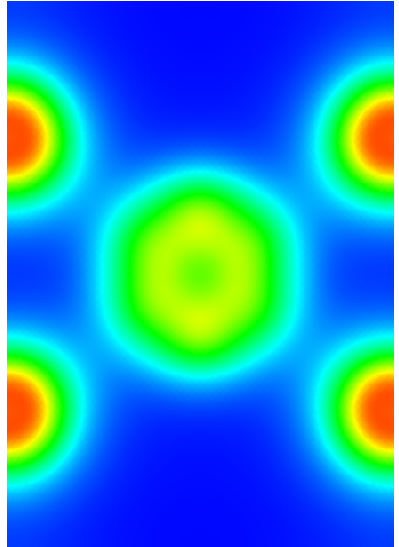

(c) $\mathrm{UN}_{2}$

4.0

3.0

2.0

1.0

0.0

(f) Scale (in $\mathrm{e} / \AA^{3}$ )

Figure 13: Valence charge density in the (100) direction of the AFM structure for $\mathrm{AcN}_{2}$ crystalline structures, where the charge distribution at the center of the slice is from Ac atom and that at face of the slice is from the $\mathrm{N}$ atom. Color scale: deep blue represents low valence charge while red represents a high concentration of valence charge.

This result contradicts the theoretical observation by $\mathrm{Lu}$ et al.[35] that predicts a thermodynamically stable structure for $\mathrm{UN}_{2}$. Our result corroborates experimental evidence by Rundle et al. [40], Tagawa et al. [16, 17] and more recent studies [46, 47] that suggests $\mathrm{UN}_{2}$ has never been prepared as a stoichiometric compound. Experimentally, it has been concluded that the correct stoichiometry may be $\mathrm{UN}_{1.75} \cdot \mathrm{UN}_{2}$ is nonstoichiometric and was assigned a hypothetical $\mathrm{CaF}$ structure on the basis of the experimental data for the actinide dinitrides with $\mathrm{N} / \mathrm{U}=1.75-1.78$ with the understanding that the non-stoichiometry arises from the presence of nitrogen vacancies in $\mathrm{UN}_{2}$. This is consistent with our theoretical observation that the nitrogen atom is the source of the instability.

A trend of reduced gap in the phonon frequency between the modes of the heavy actinide 


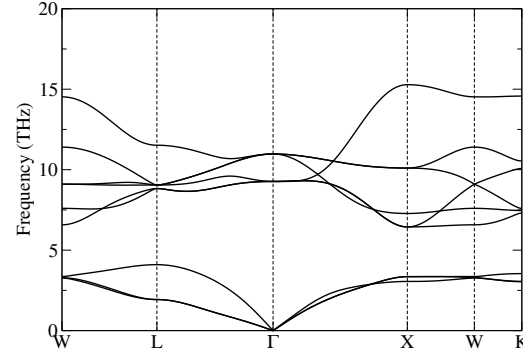

(a) $\mathrm{ThN}_{2}$

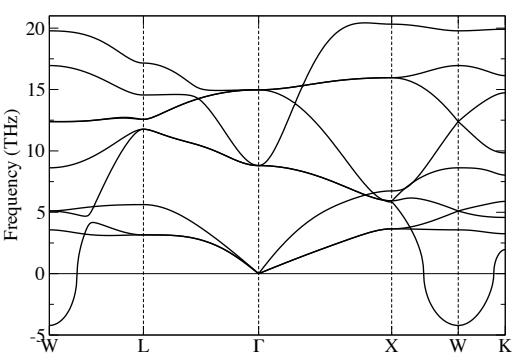

(c) $\mathrm{UN}_{2}$

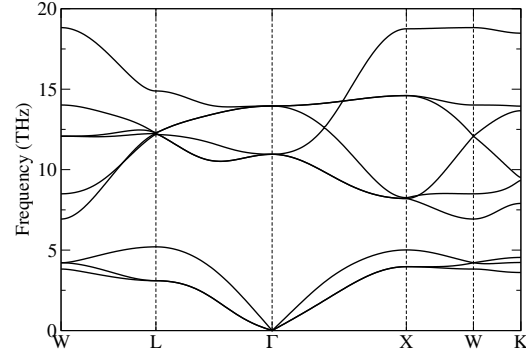

(b) $\mathrm{PaN}_{2}$

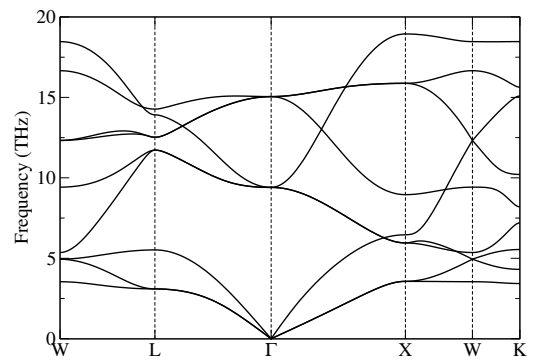

(d) $\mathrm{NpN}_{2}$

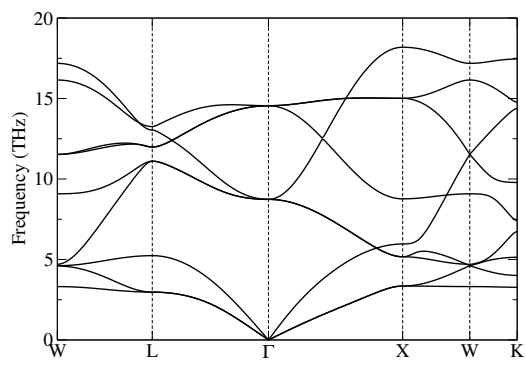

(e) $\mathrm{PuN}_{2}$

Figure 14: Phonon dispersion for $\mathrm{AcN}_{2}$ crystalline structures

metal and the light nitrogen atom is observed with increasing atomic number. Also, the difference in the frequency of nitrogen atoms compared to the actinide atoms is low compared to their $\mathrm{AcN}$ analogue. This implies that the addition of the extra nitrogen atom to the structure has led to the further stabilization of the structure.

\section{Concluding remarks}

We studied the ground state electronic, structural and mechanical properties of the AcN and $\mathrm{AcN}_{2}$ early actinide nitrides. The calculated lattice parameters with the inclusion of the Hubbard $U$ parameter agree well with available experimental data where that data exists. There is the exception of ThN, $\mathrm{PuN}$ and $\mathrm{ThN}_{2}$ compounds where GGA gives agreeable results for the actinide mononitrides and dinitrides. In general, all the actinide nitrides studied are energetically favorable, and are elastically and mechanically stable with the exception of $\mathrm{ThN}_{2}$ 
that is unstable to isotropic pressure. Notably $\mathrm{UN}_{2}$ is thermodynamically unstable and has been speculated to stabilize under $\mathrm{N}$-vacancy formation. This will be investigated in a future study.

Comparison of the calculated and experimental magnetic properties of these systems shows that the GGA $+U$ with and without SOC gives a good qualitative account of the properties compared with experiment, but not an accurate quantitative account. Therefore, going beyond DFT $+U$ is important to quantitatively determine the magnetic moments.

An analysis of the valence charge density distribution of the actinide nitrides shows the presence of ionic bonding predominantly, with the dinitrides having higher covalent character compared to the mononitrides. Also, we observe a consistent increase in the charge density distribution at the actinide sites for the actinide mononitrides and dinitrides with increasing atomic number.

All the actinide nitrides considered are metallic in nature with the exception of $\mathrm{UN}_{2}$.

\section{Acknowledgments}

We thank the University of Pretoria for financial support. KO gratefully acknowledges the support of Obodo T. Joshua and the members of the Computational and Theoretical Physics group at the University of Pretoria for useful discussions. Also, we thank Dr Jannie Pretorius and Prof. Walter Meyer for assistance with the computational hardware and software resources. Finally, NC is grateful to the National Institute for Theoretical Physics for support.

\section{References}

[1] V G Baranov, Yu N Devyatko, A V Tenishev, A V Khlunov, and O V Khomyakov. A physical model for evaluating uranium nitride specific heat. Journal of Nuclear Materials, 434:248-251, 2013.

[2] Kezhao Liu, Ren Bin, Hong Xiao, Zhong Long, Zhanglian Hong, Hui Yang, and Sheng Wu. Applied Surface Science Structural analysis of nitride layer formed on uranium metal by glow plasma surface nitriding. Applied Surface Science, 265:389-392, 2013.

[3] K O Obodo and N Chetty. First principles LDA + U and GGA + U study of protactinium and protactinium oxides: dependence on the effective U parameter. Journal of Physics: Condensed Matter, 25:145603(12), April 2013.

[4] K.O. Obodo and N. Chetty. A theoretical study of thorium titanium-based alloys. Journal of Nuclear Materials, 440:229-235, May 2013.

[5] G W Chinthaka Silva, Philippe F Weck, Eunja Kim, Charles B Yeamans, Gary S Cerefice, Alfred P Sattelberger, and Kenneth R Czerwinski. Crystal and Electronic Structures of Neptunium Nitrides Synthesized Using a Fluoride Route. Journal of The American Chemical Society, 134:3111-3119, 2012.

[6] Hiroki Shibata, Tomohito Tsuru, Tsuyoshi Nishi, Masaru Hirata, and Yoshiyuki Kaji. Thermodynamic properties of neptunium nitride : a first principles study. Journal of Nuclear Science and Technology, 49(3):328-333, 2012.

[7] Shin-ichi Fujimori, Takuo Ohkochi, Tetsuo Okane, Yuji Saitoh, Atsushi Fujimori, Hiroshi Yamagami, Yoshinori Haga, Etsuji Yamamoto, and Yoshichika Onuki. Itinerant nature of $\mathrm{U} 5 \mathrm{f}$ states in uranium mononitride revealed by angle-resolved photoelectron spectroscopy. Physical Review B, 86(23):235108, 2012.

[8] Yong Lu, Da-Fang Li, Bao-Tian Wang, Rong-Wu Li, and Ping Zhang. Electronic structures, mechanical and thermodynamic properties of ThN from first-principles calculations. Journal of Nuclear Materials, 408:136-141, January 2011.

[9] Hiroki Shibata, Tomohito Tsuru, Masaru Hirata, and Yoshiyuki Kaji. First principles study on elastic properties and phase transition of NpN. Journal of Nuclear Materials, 401(1-3):113-117, June 2010.

[10] L. Petit, A. Svane, Z. Szotek, W. M. Temmerman, and G. M. Stocks. Ground-state electronic structure of actinide monocarbides and mononitrides. Physical Review B, 80(04):045124(8), July 2009.

[11] A. O. Shorikov, A. V. Lukoyanov, M. A. Korotin, and V. I. Anisimov. Magnetic state and electronic structure of the $\delta$ and $\alpha$ phases of metallic Pu and its compounds. Physical Review B, 72(02):024458(18), July 2005.

[12] K Kurosaki, J Adachi, M Uno, and S Yamanaka. Molecular dynamics studies of actinide nitrides. Journal of Nuclear Materials, 344:45-49, September 2005. 
[13] L Black, F Miserque, T Gouder, L Havela, J Rebizant, and F Wastin. Preparation and photoelectron spectroscopy study of UNx thin films. Journal of Alloys and Compounds, 315:36-41, 2001.

[14] T Gouder, L Havela, L Black, F Wastin, J Rebizant, and Boulet P. Synthesis and electronic properties of ThN films. Journal of Alloys and Compounds, 336:73-76, April 2002.

[15] M Samsel-Czekala, E Talik, P. de V. Du Plessis, R Troć, H Misiorek, and C SuÅkowski. Electronic structure and magnetic and transport properties of single-crystalline UN. Physical Review B, 76(14):144426, 2007.

[16] Hiroaki Tagawa. Phase Relations and Thermodynamic Properties of the Uranium-Nitrogen system. Journal of Nuclear Materials, 51:78-89, 1974.

[17] Hiroaki Tagawa and Norio Masaki. X-ray and Density studies of nonstoichiometric Uranium sesquinitride. Journal of Inorganic and Nuclear Chemistry, 36:1099-1103, 1974.

[18] D Sedmidubsky and P Nova. Calculation of enthalpies of formation of actinide nitrides. Journal of Nuclear Materials, 344:40-44, 2005.

[19] Vladimir I. Anisimov, Jan Zaanen, and Ole K. Andersen. Band theory and Mott insulators: Hubbard U instead of Stoner I. Physical Review B, 44(3):943-954, 1991.

[20] A. I. Liechtenstein, V. I. Anisimov, and J. Zaanen. Density-functional theoy and strong interactions: Orbital ordering in Mott-Hubbard insulators. Physical Review B, 52(8):R5467-R5471, 1995.

[21] V I Anisimov, F Aryasctiawan, and A I Lichtenstein. First-principles calculations of the electronic structure and spectra of strongly correlated systems : the LDA + U method. Journal of Physics: Condensed Matter, 767:767-808, 1997.

[22] Raymond Atta-Fynn and Asok K. Ray. Density functional study of the actinide nitrides. Physical Review B, 76(11):115101(12), September 2007.

[23] Philippe F Weck, Eunja Kim, Naduvalath Balakrishnan, Charles B Yeamans, and Kenneth R Czerwinski. Firstprinciples study of single-crystal uranium mono- and dinitride. Chemical Physics Letters, 443:82-86, 2007.

[24] Denis Gryaznov, Eugene Heifets, and David Sedmidubsky. Density functional theory calculations on magnetic properties of actinide compounds. Physical chemistry chemical physics : PCCP, 12:12273-12278, October 2010.

[25] P. Hohenberg and W. Kohn. Inhomogeneous Electron Gas. Physical Review, 136(3B):B864-B871, 1964.

[26] G Kresse and J Furthmuller. Efficient iterative schemes for ab initio total-energy calculations using a planewave basis set . Physical Review B, 54(16):11169 (18), 1996.

[27] G Kresse and D Joubert. From ultrasoft pseudopotentials to the projector augmented-wave method. Physical Review B, 59(3):1758-1775, 1999.

[28] John P Perdew, Kieron Burke, and Matthias Ernzerhof. Generalized Gradient Approximation Made Simple. Physical Review Letters, 77(18):3865(4), 1996.

[29] Hendrik J Monkhorst and James D Pack. Special points for Brillouin-zone integration*. Physical Review B, 13(12):5188-5192, 1976.

[30] S L Dudarev, G A Botton, S Y Savrasov, C J Humphreys, and A P Sutton. Electron-energy-loss spectra and the structural stability of nickel oxide: An LSDA + U study. Physical Review B, 57(3):1505-1509, 1998.

[31] Christoph Loschen, Javier Carrasco, Konstantin Neyman, and Francesc Illas. First-principles LDA+U and GGA+U study of cerium oxides: Dependence on the effective U parameter. Physical Review B, 75(3):1-8, January 2007.

[32] C. Sevik and T. Çan. Mechanical and electronic properties of $\mathrm{CeO} 2, \mathrm{ThO} 2$, and (Ce,Th)O2 alloys. Physical Review B, 80(1):1-7, July 2009.

[33] M Fhokrul Islam and Asok K Ray. A LDA + U study of the photoemission spectra of the double hexagonal close packed phases of Am and Cm. Solid State Communications, 150(19-20):938-942, 2010.

[34] Bao-tian Wang, Hongliang Shi, Weidong Li, and Ping Zhang. First-principles LDA + U and GGA + U study of neptunium dioxide. Physical Review B, 81(045119):1-6, 2010.

[35] Yong Lu, Bao-tian Wang, Rong-wu Li, Hong-liang Shi, and Ping Zhang. Structural, electronic, mechanical, and thermodynamic properties of UN2: Systematic density functional calculations. Journal of Nuclear Materials, 410:46-51, 2011.

[36] Yvon Le Page and Paul Saxe. Symmetry-general least-squares extraction of elastic data for strained materials from ab initio calculations of stress. Physical Review B, 65(10):104104(14), 2002.

[37] R Hill. The Elastic Behaviour of a Crystalline Aggregate. Proceedings of the Physicsal Society. Section A, 65(5):349-354, 1952.

[38] Orson L Anderson. A SIMPLIFIED METHOD FOR CALCULATING THE DEBYE TEPERATURE FROM ELASTIC CONSTANTS. Journal of Physics and Chemistry of Solids, 24:909-917, 1963.

[39] K Parlinski, Z. Q. Li, and Y Kawazoe. First-Principles Determination of the Soft Mode in Cubic ZrO 2. Physical Review Letters, 78(21):4063(4), 1997.

[40] R E Rundle, N C Baenziger, A S Wilson, and R A McDonald. The Structures of the Carbides, Nitrides and Oxides of Uranium. Journal of American Chemical Society, 70(1):99-105, 1948.

[41] M S S Brooks and P J Kelly. Large Oribtal-Moment COntribution to 5f Band Magnetism. Physical Review Letters, 51(18):1708-1711, 1983. 
[42] I Shein, K Shein, and A Ivanovskii. First-principle study of B1-like thorium carbide, nitride and oxide. Journal of Nuclear Materials, 353:19-26, July 2006.

[43] L GERWARD, J STAUN OLSEN, U. BENEDICT, J P ITIE, and J. C. SPIRLET. The Crystal Structure and the Equation of State of Thorium Nitride for Pressures up to $47 \mathrm{GPa}$. Journal of Applied Crystallography, 18:339-341, 1985.

[44] R Didchenko and F P Gortsema. Magnetic and Electric Properties of Monosulfides and Mononitrides of Thorium and Uranium. Inorganic chemistry, 2:1079-1080, 1963.

[45] I.R. Shein and A.L. Ivanovskii. THORIUM COMPOUNDS WITH NON-METALS ELECTRONIC STRUCTURE, CHEMICAL BOND, AND PHYSICOCHEMICAL PROPERTIES. Journal of Structural Chemistry, 49(2):348-370, May 2008.

[46] G W Chinthaka Silva, Charles B Yeamans, Alfred P Sattelberger, Thomas Hartmann, Gary S Cerefice, and Kenneth R Czerwinski. Reaction Sequence and Kinetics of Uranium Nitride Decomposition. Inorganic chemistry, 48(22):10635-10642, 2009.

[47] Frederic Poineau and Charles B Yeamans. X-ray absorption fine structure spectroscopic study of uranium nitrides. Journal of Radioanalytical Nuclear Chemistry, 292(3):989-994, 2012. 